\title{
Towards the field binary population: influence of orbital decay on close binaries
}

\author{
C. Korntreff ${ }^{1}$, T. Kaczmarek ${ }^{2}$, and S. Pfalzner ${ }^{2}$
}

\author{
1 Jülich Supercomputing Centre, Forschungszentrum Jülich GmbH, 52425 Jülich, Germany \\ 2 Max-Planck-Institut für Radioastronomie, Auf dem Hügel 69, 53121 Bonn, Germany \\ e-mail: t.kaczmarek@mpifr-bonn.mpg.de
}

Received 2 September 2011 / Accepted 23 May 2012

\begin{abstract}
Context. Surveys of binary populations in the solar neighbourhood have discovered that the periods of G- and M-type stars are log-normally distributed in the range $0.1-10^{11}$ days. However, observations of young binary populations in various star-forming regions have instead inferred a log-uniform distribution. Some process(es) must clearly be responsible for this change in the period distribution over time. Most stars form in star clusters, so it is here that the(se) process(es) take place.

Aims. In dense young clusters, two important dynamical processes occur: i) the gas-induced orbital decay of embedded binary systems and ii) the destruction of soft binaries in three-body interactions. The emphasis in this work is on orbital decay as its influence on the binary distribution in clustered environments has largely been neglected so far.

Methods. We performed Monte Carlo simulations of binary populations to model the process of orbital decay due to friction between the gas and binary stars. In addition, the destruction of soft binaries in young dense star clusters was simulated using $N$-body modelling of binary populations.

Results. It is known that the cluster dynamics destroy the number of wide binaries, but leave short-period binaries basically undisturbed. Here we demonstrate that this result is also valid for an initially log-uniform period binary distribution. In contrast, the process of orbital decay significantly reduces the number and changes the properties of short-period binaries, leaving wide binaries largely uneffected. Until now, it has been unclear whether the short period distribution of the field has remained unaltered since its formation. We show here, that if any alteration took place, then orbital decay is a prime candidate for this task. In combination, the dynamics of these two processes, convert even an initial log-uniform distribution into a log-normal period distribution. The probability is $94 \%$ that the evolved period distribution and the observed period distribution have been sampled from the same parent distribution.

Conclusions. Our results provide a new picture for the development of the field binary population: binaries can be formed as a result of the star-formation process in star clusters with periods that are sampled from the log-uniform distribution. As the cluster evolves, short-period binaries merge to form single stars by means of gas-induced orbital decay, while the dynamical evolution in the cluster destroys wide binaries. The combination of these two equally important processes reshapes an initial log-uniform period distribution to the log-normal period distribution that is observed in the field.
\end{abstract}

Key words. binaries: general - galaxies: clusters: general - methods: numerical

\section{Introduction}

The most important observations to have shaped our current picture of the binary field population were performed in the early 1990's. Duquennoy \& Mayor (1991) found that the binary frequency of G-type stars in the solar neighbourhood is about $61 \%$ and the period distribution follows a log-normal distribution

$f(\log P)=C \exp \left\{\frac{-(\log P-\overline{\log P})^{2}}{2 \sigma_{\log P}^{2}}\right\}$,

over the period range $\sim 10^{-1}-10^{11} \mathrm{~d}$, where $P$ is the period in days $\left(\overline{\log P}=4.8 \equiv 172 \mathrm{yr}, \sigma_{\log P}=2.3\right)$ and $C$ a normalisation constant. A year later, Fischer \& Marcy (1992) analysed the binary properties of M-dwarfs in the separation range $0.04-10^{4} \mathrm{AU}$ corresponding to periods in the range $\sim 10^{-2}-10^{6} \mathrm{yr}$ and found that the period distribution of M-dwarfs in the solar neighbourhood is also log-normally distributed with a peak between $9 \mathrm{yr}$ and $270 \mathrm{yr}$, which is nearly identical to the findings for G-dwarfs. Although the observed binary frequency of M-dwarfs at about $33 \%$ is lower than that of G-dwarfs, the properties of the $\mathrm{G}$ and M-dwarf binary populations seem very similar. Observations of Raghavan et al. (2010) confirmed this picture. They determined the binary properties of nearby $(d<25 \mathrm{pc})$ solar-type stars $(\sim \mathrm{F} 6-\mathrm{K} 3)$ and found that the fraction of binary stars is $58 \%$ with a log-normal period distribution $\left(\overline{\log P}=5.03\right.$ and $\left.\sigma_{\log P}=2.28\right)$.

With an average age of a few Gyrs, the field population constitutes a dynamically evolved state. Therefore, dynamical processes have most likely changed the binary properties since the formation of these stars, and the current properties probably differ significantly from the primordial state. To understand the binary formation process, it is insufficient to know the properties of the (old) field population: we need to determine those of the primordial binary population.

Connelley et al. (2008) determined the binary properties of the very young populations in Taurus, Ophiuchus, and Orion star-forming regions (excluding the much denser Orion Nebula Cluster). They found that their observed period distribution can 
be fitted by a log-uniform distribution, which differs significantly from the log-normal distribution in the field (see also Kraus \& Hillenbrand 2007). In these sparse young star-forming regions, it is improbable that dynamical evolution has altered the period distributions in the short period since the stars were formed (see e.g. Kroupa \& Bouvier 2003). Hence, we can assume that their properties in general match the initial conditions.

In denser regions, indications of an originally log-uniform distribution have also been found. For example, the HST observations by Reipurth et al. (2007) of binaries in the Orion Nebula Cluster (ONC) demonstrate that the semi-major axis distribution deviates from the log-normal distribution and is closer to a log-uniform distribution. Hence, it seems that older binary populations have a log-normal period distribution, while the primordial distribution is likely to be log-uniform.

From the theoretical side, the binary frequency is the most well-studied binary property (e.g. Kroupa \& Burkert 2001; Kroupa \& Bouvier 2003; Kroupa et al. 2001; Pfalzner \& Olczak 2007; Marks et al. 2011; Marks \& Kroupa 2011). It also seems that the evolution of the binary frequency in the ONC depends on the initial binary frequency, whereas the evolution of the binary population does not (Kaczmarek et al. 2011). However, the binary population is not only described by the binary frequency but also by the period, mass-ratio, and eccentricity distribution.

Starting with the work of Heggie (1975), it has been realised that binaries can be dynamically disrupted by three- and fourbody interactions. Wide binaries are generally more affected by dynamical destruction than close ones. The existence of binaries wider than $10^{3} \mathrm{AU}$ in the field is still difficult to explain in models of their origin (Parker et al. 2009).

Performing $N$-body simulations, Kroupa (1995a,b) showed that to reproduce the observed log-normal distribution of the field, the initial number of wide binaries has to be significantly higher than observed, if all binaries are subject to dynamical evolution in a star cluster. This rising distribution was obtained by inverse dynamical population synthesis, i.e. inverting the effects of dynamical destruction on the period distribution (see dashed line in Fig. 1).

Parker et al. (2009) found that binaries with periods exceeding $10^{3} \mathrm{AU}$ cannot survive in the clusters that he investigated. However, binaries with semi-major axes exceeding $10^{3} \mathrm{AU}$ are observed in the field. Kouwenhoven et al. (2010) suggested that these observed wide binaries could form during the cluster dissolution. Another approach would be a different cluster type.

Here we investigate how the initial period distribution can evolve in its natal environment by considering the two different dynamical processes that inevitably affect binaries in a cluster: i) gas-induced orbital decay and ii) the dynamical destruction of binaries in encounters.

The influence of orbital decay has so far only been investigated for isolated binary systems Stahler (2010). Orbital decay takes place during the earliest stages of star formation, when the stars are still embedded in the gas. Here the interaction of binaries with the gas leads to the excitation of waves in the surrounding gas. The energy transfer from the binary to the gas leads to orbital decay (Stahler 2010). Here we investigate how orbital decay changes the period distribution in a cluster environment.

This paper is structured in the following way: in Sect. 2, the cluster setup is explained. Section 3 describes the orbital decay process in the gas-embedded phase. We demonstrate in Sect. 4 that a combination of the orbital decay with the dynamical destruction (Kaczmarek et al. 2011) naturally transforms a lognormal birth period distribution into a log-uniform period distribution. Our conclusions are given in our final section.

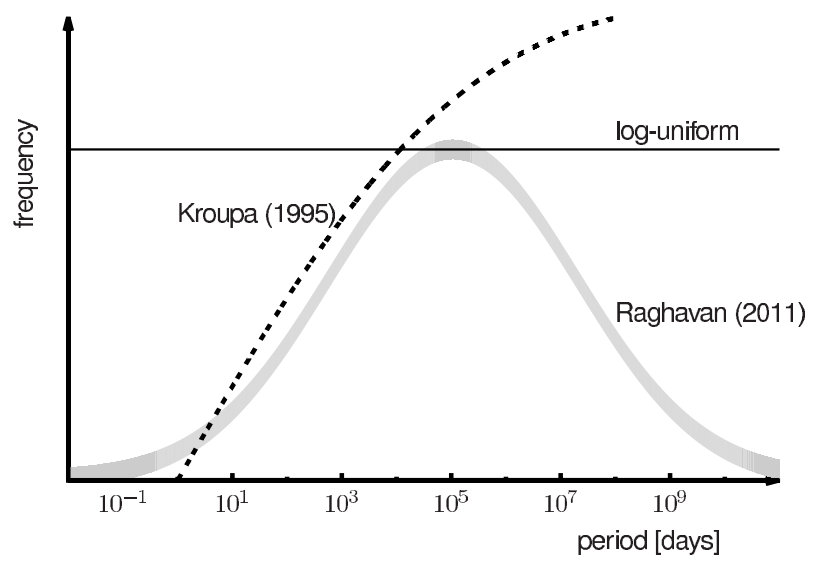

Fig. 1. Schematic picture of possible initial period distributions. The thick grey curve displays the log-normal fit to the data of Raghavan et al. (2010), the thick dashed line the Kroupa (1995a), and the solid line a log-uniform period distribution, which is observed for many young starforming regions (see text for references).

\section{Cluster setup}

The processes of orbital decay and dynamical disruption inevitably take place as soon as the binary population forms in a dense star-cluster environment. Both result from interactions between stars in a dense environment. As most stars form in cluster environments (Lada \& Lada 2003), it can be anticipated that the field binary population that we observe today was - at least to same extent - shaped by these processes.

In this study, we chose the Orion Nebula Cluster (ONC) as our model cluster. It is probably a typical star-forming region and has the advantage that many parameters of both the cluster structure and the binary population have been well-observed (e.g. Jones \& Walker 1988; Prosser et al. 1994; Hillenbrand 1997; Hillenbrand \& Hartmann 1998; O’Dell et al. 2009; Preibisch et al. 1999; Köhler et al. 2006; Reipurth et al. 2007). The ONC's radial density profile declines in its outer parts as $\rho_{\text {present }} \propto r^{-2}$ (Jones \& Walker 1988; Hillenbrand 1997) and is much flatter $\left(\rho_{\text {core }} \propto r^{-0.5}\right)$ within $R_{\text {core }} \approx 0.2$ pc. The dynamical evolution of our model cluster significantly changes the stellar density profile during the first Myr, which is the estimated age of the ONC (Hillenbrand 1997). Hence, we adopted the following initial density profile

$\rho_{\text {star }}(r) \propto\left\{\begin{array}{cc}\left(r / R_{\text {core }}\right)^{-2.3}, & 0<r \leq R_{\text {core }} \\ \left(r / R_{\text {core }}\right)^{-2.0}, & R_{\text {core }}<r \leq R \\ 0, & R<r \leq \infty .\end{array}\right.$

Here we chose $R_{\text {core }}=0.2 \mathrm{pc}$ and a cluster size of $R=2.5 \mathrm{pc}$ to model the ONC. The adopted model starts from a situation where all stars have already been formed. In dynamical equilibrium, the initial radial density profile (Eq. (2)) of our model cluster evolves towards the currently observed one over a time span of about 1 Myr (Olczak et al. 2010). However, other initial conditions could result in the same profile (Kroupa 2000; Allison et al. 2009).

To mimic the often observed mass-segregation in young dense clusters, the most massive binary system is initially placed at the cluster centre and the next three most massive stars at random positions within a sphere of radius $R=0.6 R_{\mathrm{hm}}$ around the cluster centre, where $R_{\mathrm{hm}}$ is the cluster half-mass radius. In implementing this, we followed the suggestions of Bonnell \& Davies (1998), who found that the observed mass segregation (Hillenbrand \& Hartmann 1998) of the ONC is unlikely to be 
the result of the dynamical evolution of the cluster but has to be primordial, if the cluster is initially in virial equilibrium. We note that if the cluster were to be in a subvirial state, probably no primordial mass segregation would be required (Allison et al. 2009; Olczak et al. 2011).

All stellar systems are initially created as binaries with system masses sampled from the initial mass function (IMF) suggested by Kroupa (2007, Eqs. (19) and (20)). We limited our system mass to $M_{\mathrm{sys}}=M_{\mathrm{s} 1}+M_{\mathrm{s} 2}$ to $\left[0.08 M_{\odot}, 50 M_{\odot}\right]$, where $M_{\mathrm{s} 1}$ and $M_{\mathrm{s} 2}$ are the masses of the stars constituting a binary system. The upper mass limit corresponds to the mass of the most massive binary system in the ONC. The lower limit is the hydrogen burning limit, thus the margin between sub-stellar and stellar objects.

Ideally we would like to use the observed initial properties of binaries in very young embedded clusters. We later demonstrate that embedded clusters younger than $100000 \mathrm{yr}$ would be ideal, because at such a young age the binary population is close to its primordial state. However, it is very difficult to observe clusters at such a young age. Observations of binaries in dense embedded clusters face a number of observational difficulties such as extinction, crowding, too low spatial resolution etc. In addition, the number of stars that could be observed would be very low, making statistical statements impossible. As forming clusters have an age spread (1-3 Myr), it should be possible to use only the youngest stars in a forming cluster to deduce the primordial state. However, the age determination of these young stars is problematic. Alternatively, one can assume that low density clusters are relatively unaffected by the processes described here and are therefore close to their primordial state. Observations of the primordial state being unavailable, we considered the work of Kouwenhoven et al. (2007) based on observations of the lowdensity Scorpius OBII cluster. Choosing the semi-major axis and mass ratio distribution from an $\mathrm{OB}$ association with an age of (5-20) Myr (Kouwenhoven et al. 2007), such as Scorpius OBII, seems at first glance unusual, as at that age the binary population might have already been processed. However, the low stellar density of Scorpius OBII combined with the lack of surrounding gas implies that the cluster has been processed neither by orbital decay nor dynamical destruction, as mentioned before.

The primordial semi-major axis distribution was chosen to be $\log$-uniform $f_{a}(a) \propto \mathrm{d} N / \mathrm{d} a \propto a^{-1}$ where $a \in$ [0.02 AU, $10000 \mathrm{AU}]$. As a primordial mass ratio distribution, we started with $f_{q}(q) \propto q^{0.4}$ and $q=M_{\mathrm{s} 2} / M_{\mathrm{s} 1} \in[0,1]$.

Another justification of our choice of initial semi-major axis distribution is that the log-uniform distribution is a straightforward distribution to adopt that requires strong processing in order to obtain the log-normal field distribution. This distribution was used here to test, whether even this extreme assumption leads to the log-normal distribution observed in the field. This does not rule out that one obtains a similar result to that described in the following with different initial conditions, namely that there are fewer wide and/or close binaries.

In the following, we describe how the aforementioned model was used to study the effect of the gas-induced orbital decay of binaries in star clusters.

\section{Gas-induced orbital decay}

In its early embedded phases, clusters consist of the already formed stars and a large gas component from which additional stars can potentially form. These stars remain embedded in their natal gas cloud until it is removed by strong winds, the radiation of the massive stars, and supernova explosions. During this embedded phase, dynamical friction occurs between the stars and binary systems and the ambient gas. Binary systems induce spiral waves in the gas, leading to energy and angular momentum losses, a shrinkage of the binary orbit, and possibly the merging of the two stars. Stahler (2010) derived an analytic expression for the temporal development of the separation $a_{\text {tot }}$ for a single isolated binary system on a circular orbit. The separation diminishes according to a gas-induced orbital decay with time as

$a_{\mathrm{tot}}=a_{0}\left(1-\frac{t}{t_{\mathrm{c}}}\right)$

where $a_{0}$ is the initial binary separation and $t_{\mathrm{c}}$ is the so-called coalescence time, which is given by

$t_{\mathrm{c}}=\frac{15}{32 \pi} \frac{(1+q)^{2}}{q} \frac{c_{\mathrm{s}}^{5}}{\rho_{0}} \frac{a_{0}}{G^{3} M_{\mathrm{sys}}^{2}}$,

where $G$ is the gravitational constant, $c_{\mathrm{s}}$ the sound speed, $q$ the mass ratio, and $M_{\text {sys }}$ the system mass of the binary system.

Stahler (2010) assumed in his derivation that gas interactions close to the stars themselves can be neglected. The binary system, which is represented by an oscillating gravitational potential, torques the nearby gas and produces outgoing acoustic waves. These waves transport angular momentum from the binary to the surrounding gas. Thus, the orbit of the binary decays as long as the gas density is high enough. Equation (3) is only valid for radial distances

$a_{\mathrm{tot}}<r^{\max }=\frac{G M_{\mathrm{sys}}^{\min }}{2 c_{\mathrm{s}}^{2}}=111 \mathrm{AU}\left(\frac{M_{\mathrm{sys}}^{\mathrm{min}}}{M_{\odot}}\right)\left(\frac{c_{\mathrm{s}}}{2 \mathrm{~km} \mathrm{~s}^{-1}}\right)^{-2}$,

between the two stars constituting the binary system.

To analyse the effect of the gas-induced orbital decay of binary systems on the period distribution, we applied Eq. (3) to the entire population of a young ONC-like star cluster. Starting from a cluster with the initial conditions given in Sect. 2, the temporal development of the binary population is followed for different gas density models.

Only when a binary system fulfills the restriction given by Eq. (5) is the orbital decay calculated, otherwise the orbit remains unaltered. This means, for example, that for a system mass of $0.08 M_{\odot}, 1 M_{\odot}$, and $50 M_{\odot}$ the orbital decay is only calculated for binary systems that have separations $r<8.88 \mathrm{AU}$, $r<111 \mathrm{AU}$, and $r<5550 \mathrm{AU}$, respectively. As in Stahler's modelling of the orbital decay, we adopted the simplification of treating all orbits as circular. Future work should also consider elliptical orbits.

For gas-induced decay, the gas density distribution in the cluster is of vital importance. Here we assume an isothermal gas density that follows the stellar density distribution. To prevent the distribution from diverging at the centre, the density is kept constant at a value $\rho_{\max }$ inside the cluster core radius $R_{\text {core }}=0.2 \mathrm{pc}$. Outside this area, the density decreases isothermally. Thus, the isothermal gas density distribution can be described by the equation

$\rho_{\text {gas }}(r)=\rho_{\max }\left\{\begin{array}{c}1, \quad r<R_{\text {core }} \\ \left(R_{\text {core }} / r\right)^{2}, R_{\text {core }}<r<R_{\text {cluster }} .\end{array}\right.$

For an isothermal density distribution $\rho_{\text {gas }}(r)=c_{\mathrm{s}}^{2} / 2 \pi G r^{2}$ (e.g. Binney \& Tremaine 1987, Eq. (4.123)), the sound speed is given by

$c_{\mathrm{s}}=\sqrt{2 \pi G \rho_{\mathrm{gas}}(r) r^{2}}$. 
Table 1. Percentage of merged binary systems for different sound speeds and maximum densities after $1 \mathrm{Myr}$.

\begin{tabular}{lccc}
\hline \hline $\begin{array}{l}\text { Sound speed } \\
\text { maximum density }\end{array}$ & $0.15 \mathrm{~km} \mathrm{~s}^{-1}$ & $0.49 \mathrm{~km} \mathrm{~s}^{-1}$ & $1.54 \mathrm{~km} \mathrm{~s}^{-1}$ \\
\hline $10^{4} \mathrm{~cm}^{-3}$ & $37.4 \%$ & $2.8 \%$ & $0.1 \%$ \\
$10^{5} \mathrm{~cm}^{-3}$ & $54.9 \%$ & $12.0 \%$ & $0.2 \%$ \\
$10^{6} \mathrm{~cm}^{-3}$ & $69.2 \%$ & $28.7 \%$ & $1.2 \%$ \\
\hline
\end{tabular}

Although the density distribution (Eq. (6)) is not isothermal over the whole parameter range, we approximated the sound speed as

$c_{\mathrm{s}}=\sqrt{2 \pi G \rho_{\max } r_{\text {core }}^{2}}$.

The resulting sound speeds for distributions with $\rho_{\max } \in$ $\left[10^{4}, 10^{6}\right] \mathrm{cm}^{-3}$ lie between the sound speed of an ideal gas at $10 \mathrm{~K}, c_{\mathrm{s}}=0.2 \mathrm{~km} \mathrm{~s}^{-1}$, and the observed value in infrared dark clouds (Sridharan et al. 2005) $c_{\mathrm{s}}=$ [1.0-2.2] $\mathrm{km} \mathrm{s}^{-1}$. A even more reliable approach would be a time-dependent density $\rho(r, t)$ and sound speed $c_{\mathrm{s}}(r, t)$, which has not yet been observed. Variations of the sound speed manifest themselves in the coalescence time (Eq. (4)).

If the separation becomes smaller than the radius of both stars $a_{\mathrm{tot}}<R_{\mathrm{s} 1}+R_{\mathrm{s} 2}$ assuming a main-sequence mass-radius relationship (Binney \& Merrifield 1998, p. 110)

$\left(\frac{R_{\mathrm{s} 1 / 2}}{R_{\odot}}\right) \approx\left(\frac{M_{\mathrm{s} 1 / 2}}{M_{\odot}}\right)^{0.7}$,

a binary system is treated as a "merged" system. This is clearly a strong simplification, because the merging of stars is a far more complex process that depends on a variety of parameters such as the presence of a surrounding disc, the eccentricity of the orbit, mass transfer, and the conditions of the molecular cloud in which the stars are embedded. However, including all these processes was beyond the aims of the current paper. Here, all processes at such small distances were excluded under the assumption that the two stars in such cases might possibly merge.

We first analysed how the binary population parameters change in general owing to orbital decay. Therefore, a series of simulations with three different gas densities in the range of $\left[10^{4} \mathrm{~cm}^{-3}, 10^{6} \mathrm{~cm}^{-3}\right]$ were performed corresponding to moderate gas densities observed in star-forming regions (Padmanabhan 2001). For the three densities $10^{4} \mathrm{~cm}^{-3}, 10^{5} \mathrm{~cm}^{-3}$, and $10^{6} \mathrm{~cm}^{-3}$, the sound speeds are $0.15 \mathrm{~km} \mathrm{~s}^{-1}, 0.49 \mathrm{~km} \mathrm{~s}^{-1}$, and $1.54 \mathrm{~km} \mathrm{~s}^{-1}$, respectively (Eq. (8)). The results, which are summarised in Table 1, show the merger rates for all combinations of gas densities and sound speeds. As expected, the percentage of possibly merged binary systems after 1 Myr (Table 1) depends on the maximum density of the isothermal density distribution (Eq. (6)). The overall reduction in the number of binaries could vary at a constant sound speed by a factor of 2-4 if the density were to change by one order of magnitude. Keeping the density constant, one can see that the percentage of merged systems is sensitive to the choice of sound speed.

Additionally, the degree of orbital decay depends on the gas density distribution. Here we considered only the case of a temporary constant and spatially isothermal gas density distribution as this most closely reproduces the observed period distribution and seems a realistic assumption for our studied case of an ONC-type cluster.

In the following, we describe how an isothermal gas density distribution with $\rho_{\max }=10^{5} \mathrm{~cm}^{-3}$ was used for the example of an ONC-like cluster.

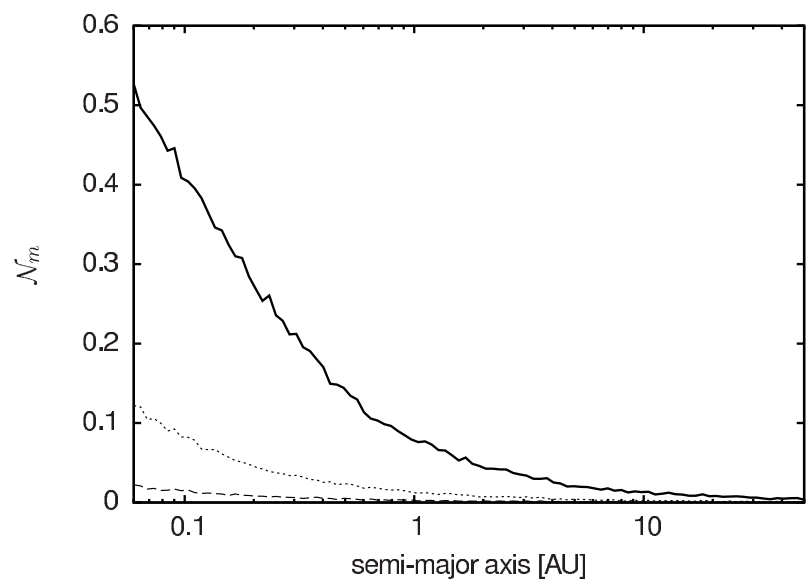

Fig. 2. The number of merged binaries relative to the initial number of binaries after $0.01 \mathrm{Myr}$ (dashed), $0.1 \mathrm{Myr}$ (dotted) and $1 \mathrm{Myr}$ (solid) for a binary distribution embedded in an $r^{-2}$ gas density distribution with $\rho_{\max }=10^{5} \mathrm{~cm}^{-3}$ and a sound speed of $0.49 \mathrm{~km} \mathrm{~s}^{-1}$.

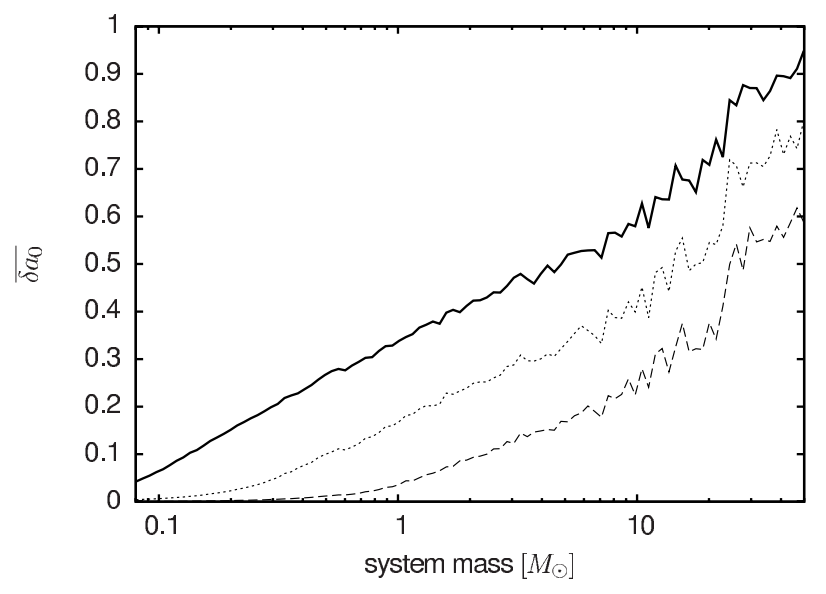

Fig. 3. Mean relative semi-major axis change as a function of the system mass for a binary distribution embedded in an $r^{-2}$ gas density distribution with $\rho_{\max }=10^{5} \mathrm{~cm}^{-3}$ and a sound speed of $0.49 \mathrm{~km} \mathrm{~s}^{-1}$ after $0.01 \mathrm{Myr}$ (dashed), 0.1 Myr (dotted), and $1 \mathrm{Myr}$ (solid).

In Fig. 2, the number of merged binaries $N_{m}$ relative to the initial number of binaries $N_{\text {tot }}, \mathcal{N}_{m}=N_{m} / N_{\text {tot }}$ is plotted as a function of the initial semi-major axis of the merged binary systems. The binaries with small semi-major axes merge to a greater degree than wider binaries. While binaries with initial semi-major axis of 10 AU rarely merge, $40 \%$ of all binaries with initial semimajor axis of $0.1 \mathrm{AU}$ coalesce after $1 \mathrm{Myr}$ (solid line).

The resulting semi-major axis change also strongly depends on the system mass $M_{\text {sys }}$. Figure 3 shows the mean relative semimajor axis change $\overline{\delta a_{0}}=[a(0)-a(t)] / a(0)$ as a function of the system mass. This illustrates that the semi-major axis of binaries with a high system mass shrinks faster and to a greater degree than the semi-major axis of binaries of a lower system mass. The shorter coalescence time for massive systems (see Eq. (4)) results from the greater angular momentum and energy loss of high-mass binaries caused by dynamical friction (Eq. (51) in Stahler 2010). The average separation between binary systems with $M_{\text {sys }}>10 M_{\odot}$ dwindles to smaller than half its initial value after 1 Myr. In addition, the location of the binary system inside the cluster influences the coalescence time. For a maximum density of $10^{5} \mathrm{~cm}^{-3}$ in the cluster core, the density drops to $6.4 \times 10^{2} \mathrm{~cm}^{-3}$ at $2.5 \mathrm{pc}$. Therefore, the coalescence time of 


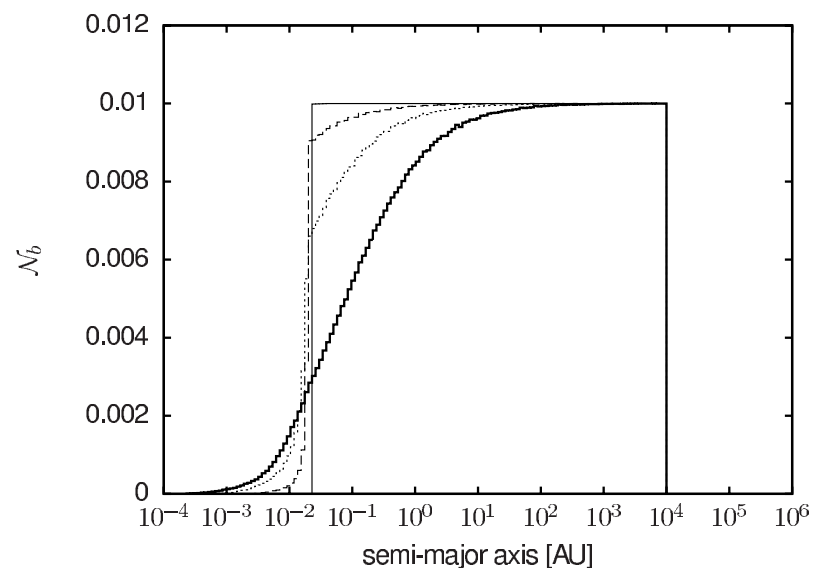

Fig. 4. Chosen initial semi-major axis distribution (thin solid line) and effect of the gas-induced orbital decay on a semi-major axis distributions embedded in an $r^{-2}$ gas density distribution with $\rho_{\max }=10^{5} \mathrm{~cm}^{-3}$ and a sound speed of $0.49 \mathrm{~km} \mathrm{~s}^{-1}$ after $0.01 \mathrm{Myr}$ (dashed), $0.1 \mathrm{Myr}$ (dotted), and 1 Myr (thick solid).

a binary system with $M_{\text {sys }}=10 M_{\odot}$ drops from $0.23 \mathrm{Myr}$ at the cluster centre to $35.95 \mathrm{Myr}$ at $2.5 \mathrm{pc}$. Considering this, the effect of orbital decay might even boost the mass segregation of the cluster.

The overall effect of the gas-induced orbital decay of the binaries in the cluster is to reshape the period distribution by pushing binaries to tighter orbits, which can even lead to the merging of a binary. This can be seen in Fig. 4, which shows the relative numbers of binary systems $\mathcal{N}_{\mathrm{b}}=N_{\mathrm{b}}(t) / N_{\mathrm{b}}(0)$ as a function of the semi-major axis after $0 \mathrm{Myr}$ (thin solid line), $0.01 \mathrm{Myr}$ (dashed line), 0.1 Myr (dotted line), and $1 \mathrm{Myr}$ (thick solid line) for a binary distribution embedded in a $r^{-2}$ gas density distribution with $\rho_{\max }=10^{5} \mathrm{~cm}^{-3}$ and a sound speed of $0.49 \mathrm{~km} \mathrm{~s}^{-1}$. Since the orbital decay is more rapid for tighter binaries (see Eq. (4)), binaries in the left part of the period semi-major axis distribution are first altered leading to a depopulation of binaries with semimajor axes between $0.02 \mathrm{AU}$ and $1 \mathrm{AU}$ and the formation of a tail for semi-major axes shorter than 0.02 AU after 0.01 Myr. As time proceeds, binaries with even larger orbits are affected until the orbital decay process stops when the gas is expelled from the cluster. At this point, the process of orbital decay alone is responsible for the binary frequency in the cluster declining from its initial value of $100 \%$ to $88 \%$ by means of the merging of very tight binaries.

\section{Comparison with observations}

We compare our aforementioned results with observations. To do so, we converted our semi-major axis distributions into period distributions and re-binned our data according to Raghavan et al. (2010). Their observations were limited to F6-K3 dwarf stars, which roughly correspond to binary systems with primary masses $M_{\mathrm{s} 1} \in\left[0.5 M_{\odot}, 1.5 M_{\odot}\right]$.

Figure 5a shows the resulting initial period distributions as thin solid lines and the final period distributions as thick solid lines for the process of orbital decay. In accordance with the results for the semi-major axis distribution, the orbital decay (Sect. 3) reduces the number of short period binaries. Using the above model, we studied the influence of the gas on the binary system, but neglected the dynamical interactions between the binaries and other stellar components in the cluster. It is known that dynamical interactions destroy long-period binaries. The
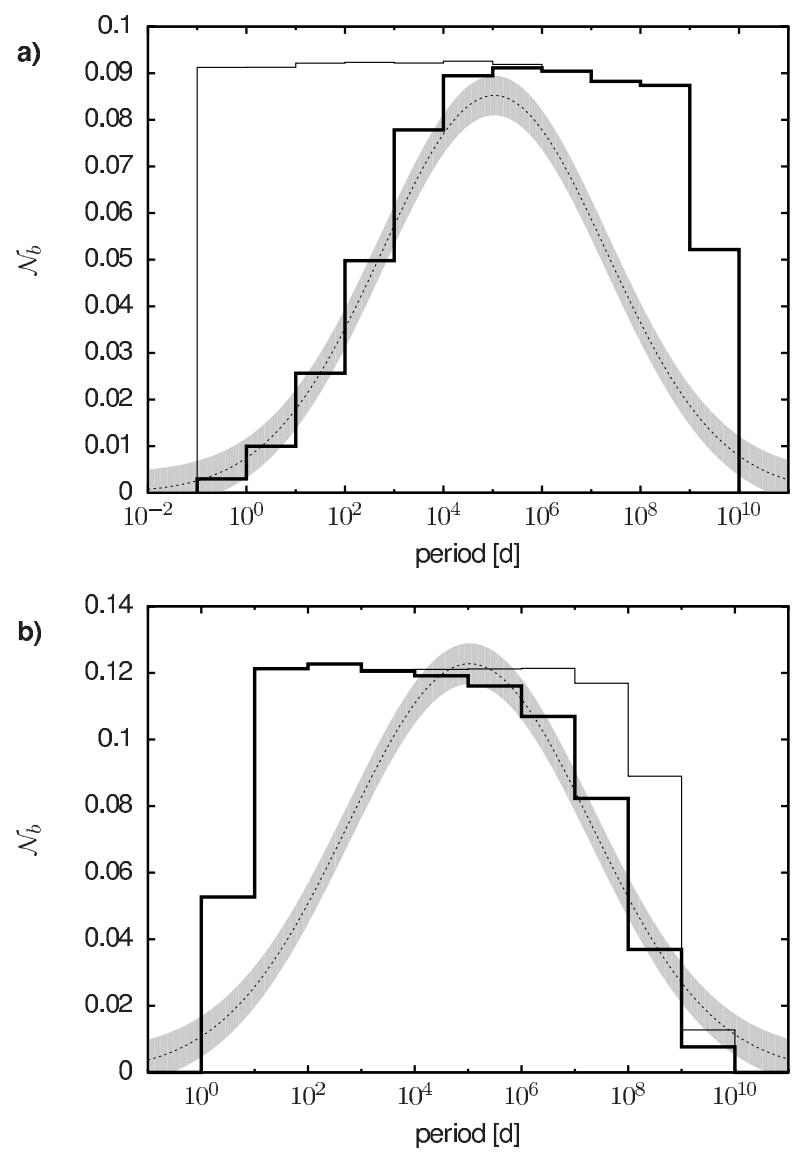

Fig. 5. a) Chosen initial period distribution (thin solid line) and effect of the gas-induced orbital decay on period distributions of binaries embedded in an $r^{-2}$ gas density distribution with $\rho_{\max }=10^{5} \mathrm{~cm}^{-3}$ and a sound speed of $0.49 \mathrm{~km} \mathrm{~s}^{-1}$ after 1 Myr (thick solid line), compared with a Gaussian fit (dotted line) to the observations by Raghavan et al. (2010). b) Same as in a), with results from the NBody6 simulations.

question arises of whether there is any region in the period distribution that is affected significantly by both of these processes.

To attempt to answer this, we wished to illustrate the dynamical impact of the cluster evolution on the binary population in the absence of the "gas-effect" described above. We therefore defined all clusters as not containing any gas and being initially in virial equilibrium. We investigated only the first 3 Myr. As only the most massive stars evolve significantly during this time, stellar evolution was not considered in the simulations. To improve statistics, we simulated 50 realisations of these clusters and averaged these results. For more details on the numerical method, we refer to Kaczmarek et al. (2011).

To provide comparable initial conditions as in Sect. 3, all simulations were set up as described in Sect. 2. The formal initial binary frequency was chosen to be $100 \%$ i.e. each star was generated as part of a binary system. However, the resulting initial binary frequency was always lower as a consequence of the setup procedure. All particles in the simulations were created as binaries initially with semi-major axes sampled from the log-uniform distribution. Afterwards, these binaries were placed randomly in the cluster independently of the semi-major axis. Therefore, binaries with separations larger than the local mean separation often already have a closer partner, so that there are fewer large-separation binaries than expected for this set up.

Figure $5 \mathrm{~b}$ shows the period distribution of the simulated cluster initially (thin solid line) and at the age of $3 \mathrm{Myr}$ (solid line), as well as the Gaussian fit (dotted line) to the observations. It can 
be seen that dynamical evolution of the binary population in a star cluster mainly destroys wide binaries, but does not affect tight binaries. These wide binaries are destroyed in threeand four-body encounters where a perturber transfers some of its kinetic energy to the binary system so that after the interaction, the binding energy of the binary becomes positive (Heggie 1975). However, this process is only effective if the binary is not too strongly bound, which implies that predominantly wide binaries are affected. Nevertheless, after $3 \mathrm{Myr}$ a small number of wide binaries still exist. Our results yield no definite conclusion about the origin of these wide binaries. They are either remnants of the initial binary population or produced by dynamical processes. Although we also performed a large number of simulations, the results for the wide binaries still suffer from low number statistics.

Similar results were obtained by e.g. Kroupa (1995a) and Kroupa et al. (2001) using initial period distributions with a larger number of initial wide binaries than used here (dashed line in Fig. 1). We note that, using a log-normal distribution with a initially lower number of wide binaries (thick grey line in Fig. 1), Parker et al. (2009) inferred that there is a deficiency of wide binaries compared to the field. If the initial binary distribution were log-normal, this would require wide binaries to be formed by other processes as suggested for example by Kouwenhoven et al. (2010) and Moeckel \& Bate (2010). However, apart from the wide binaries, the overall result does not seem to depend on the initial distribution. In our study here, we applied a log-uniform period distribution as for the investigation of the orbital decay. The treatment was otherwise very similar to previous work.

The destruction of the wide binary systems is accompanied by a reduction in the binary frequency by $13 \% \pm 2 \%$ after $3 \mathrm{Myr}$ where the error is given by the standard deviation among the 50 realisations. However, this represents only a lower limit to the destruction of binaries by dynamical interactions. As mentioned above, although all stars are intended to be initially part of a binary, a certain fraction of all binaries - dominantly the wide ones - are "disrupted" during the setup process. Treating these as being destroyed by the cluster dynamics results in an upper limit of $26 \% \pm 2 \%$. In summary, the effect of the dynamical destruction of wide binaries is a reduction of $\approx 13 \%-26 \%$ in the binary frequency during the first $3 \mathrm{Myr}$. Comparing this to the $12 \%$ loss of binaries by mergers caused by orbital decay, this means that both processes are of equal importance, at least for the considered case.

In the following, we specify the maximum effective period for the orbital decay $P_{\text {orb }}$ and the minimum effective period for the dynamical destruction $P_{\text {dyn }}$ at which at least $3 \%$ of the binaries are affected by the corresponding processes (see Fig. 6). Since the resulting values are $P_{\text {orb }}=5.5 \times 10^{4}$ days and $P_{\text {dyn }}=1.1 \times 10^{5}$ days, it follows that $P_{\text {orb }}<P_{\text {dyn }}$. Hence, there is no period range where both processes simultaneously play a role. Separating the two process as done here, does not take into account binaries that exchange partners and become more strongly bound. However, testing this for the first $3 \mathrm{Myr}$ of the cluster development considered here, we found that only in $\ll 1 \%$ of all cases does binary harding lead to a transgression into the regime where orbital decay takes place. This means that both processes can be treated separately (see Fig. 6) in a additive way.

To determine the likelihood that our simulated period distributions correspond to the observed field population, we performed $\chi^{2}$-tests as described by Press et al. (2007)

$\chi^{2}=\sum_{i=1}^{N} \frac{\left(R_{i}-S_{i}\right)^{2}}{R_{i}+S_{i}}$,

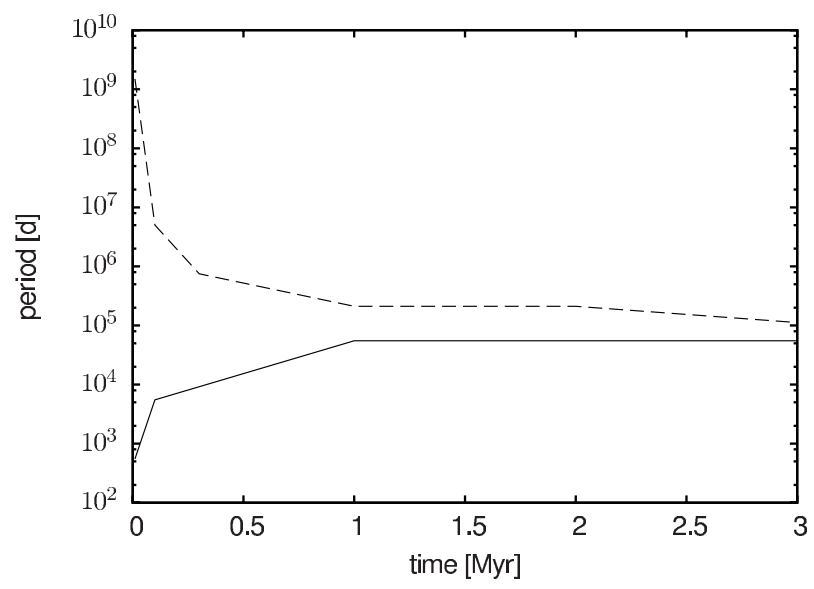

Fig. 6. Evolution of $P_{\mathrm{dyn}}$ (upper line) and $P_{\mathrm{orb}}$ in the first $3 \mathrm{Myr}$ of cluster evolution.

Table 2. $\chi^{2}$-test results calculated for the initial and final theoretical period distributions and those observed by Raghavan et al. (2010).

\begin{tabular}{l|cc|cr}
\hline \hline & \multicolumn{3}{|c}{ Orbital decay } & \multicolumn{3}{c}{$N$-body dynamics } \\
\hline & \multicolumn{4}{|c}{ complete period range } \\
& $\chi^{2}$ & $Q\left(\chi^{2} \mid v\right)$ & $\chi^{2}$ & $Q\left(\chi^{2} \mid v\right)$ \\
Initial & 71.5 & $2.3 \times 10^{-11}$ & 23.6 & 0.009 \\
Final & 36.4 & $7.2 \times 10^{-5}$ & 22.4 & 0.013 \\
\hline & \multicolumn{4}{|c}{ Adopted period ranges } \\
& $\chi^{2}$ & $Q\left(\chi^{2} \mid v\right)$ & $\chi^{2}$ & $Q\left(\chi^{2} \mid v\right)$ \\
Initial & 45.7 & $1.1 \times 10^{-8}$ & 6.6 & 0.171 \\
Final & 3.2 & 0.67 & 0.9 & 0.92 \\
\hline
\end{tabular}

Notes. The first four column block shows the results of tests of the complete Raghavan et al. (2010) period distribution ranging from $10^{-1}$ to $10^{10} \mathrm{~d}$. The second four column block shows tests of the left part of the Raghavan et al. (2010) period distribution $\left(10^{-1}-10^{5} \mathrm{~d}\right)$ for the orbital decay distributions and the right part of the Raghavan et al. (2010) period distribution $\left(10^{-5}-10^{10} \mathrm{~d}\right)$.

where $N$ is the number of bins, and $R_{i}$ and $S_{i}$ are the two distributions to be tested. This is only applicable if $\sum_{i} R_{i}=\sum_{i} S_{i}$. To warrant this, we scaled our probability distributions to the total number of binaries observed by Raghavan et al. (2010). Afterwards, the $\chi^{2}$-probability function $Q\left(\chi^{2} \mid v\right)$ is used to calculate the probability that both distributions were sampled from the same underlying distribution.

Table 2 shows the results of $\chi^{2}$-tests for the Raghavan et al. (2010) period distribution and the simulated one. The probability that the initial period distribution and the observed period distribution of the field were sampled from the same underlying distribution is obviously negligible (probabilities of $3.5 \times 10^{-7}$ and $\left.4.0 \times 10^{-3}\right)$. Similarly, considering only one of the two processes "orbital decay" or "dynamical interaction" alone results in (higher, but still) low probabilities. Restricting the $\chi^{2}$-test to the proper period ranges $\left(10^{-1}-10^{5} \mathrm{~d}\right.$ for the orbital decay and $10^{5}-10^{10} \mathrm{~d}$ for the cluster dynamics) yields much more robust results - both the orbital decay and the cluster dynamics result in $Q\left(\chi^{2} \mid v\right)$ values of $67 \%$ and $92 \%$, respectively, indicating the extremely good agreement of our simulated period distributions and the observations by Raghavan et al. (2010). A superposition of the distribution of periods $P<10^{5} \mathrm{~d}$ depleted by orbital decay and the distribution of periods $P>10^{5} \mathrm{~d}$ resulting from the dynamical destruction is shown as a thick solid line in Fig. 7. Performing the $\chi^{2}$-test on this complete distribution over the entire period range results in a $Q\left(\chi^{2} \mid v\right)$-value of $94.1 \%$. This 


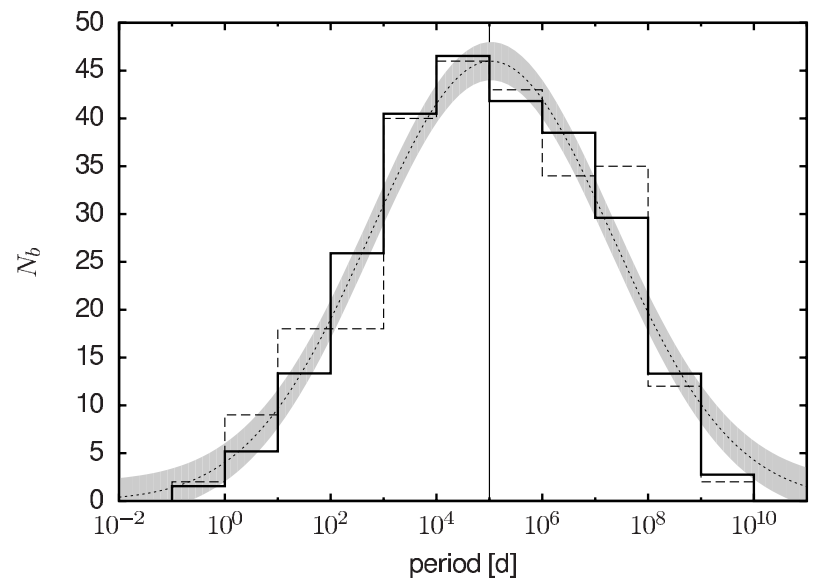

Fig. 7. Comparison of the period distributions resulting from the orbital decay of embedded binaries and the dynamical destruction (thick solid line) with the observations of Raghavan et al. (2010) (dashed line). Additionally, the log-normal fit by Raghavan et al. (2010) is shown as a short-dashed line.

clearly demonstrates that together these two processes naturally reshape a log-uniform distribution to a log-normal distribution as observed in the field today.

\section{Conclusions}

For the first time, we have demonstrated that the gas-induced orbital decay of binaries in the embedded cluster phase significantly changes the properties of the short-period binary distribution. It turns out that this process is of equal importance to the development of the initial binary population, as part of the wellstudied process of dynamical evolution, at least for ONC-like clusters.

Gas-induced orbital decay not only changes the binary properties, but can even lead to the mergers of binaries, creating a more massive single star. The likelihood of these mergers depends on the density profile of the gas and both the separation and the mass of the binary system. For example, while binaries with semi-major axis of $10 \mathrm{AU}$ rarely merge after $1 \mathrm{Myr}$ in a cluster with a maximum gas density of $10^{5} \mathrm{~cm}^{-3}, 40 \%$ of all binaries with a semi-major axis of $0.1 \mathrm{AU}$ do so. In general, the separation between binary systems with $M_{\text {sys }}>10 M_{\odot}$ shrinks on average to less than half its initial value after $1 \mathrm{Myr}$.

Orbital decay only changes the period distribution of short period binaries. However, long-period binaries are affected by dynamical destruction caused by the interaction of the stars in a dense cluster environment. Our simulations show that intermediate-period binaries are nearly unaffected by either of these two processes (see Fig. 8).

For an ONC-like cluster, we found that for these G-type primary stars the orbital decay due to the interaction of the binaries with the ambient gas basically affects only binaries with periods shorter than $5 \times 10^{4} \mathrm{~d}$ corresponding to separations closer than $\approx 36$ AU. In contrast, dynamical interactions only destroy binaries with periods longer than $10^{5}$ d (i.e. $\approx 53 \mathrm{AU}$ ), meaning that there is no region in the period distribution that is influenced by both effects. It is therefore possible to investigate the two processes separately and combine our results.

Perhaps the most striking result of this investigation is, that these two processes transform a primordial log-uniform into a log-normal period distribution, on a realistic timescale. The dis-

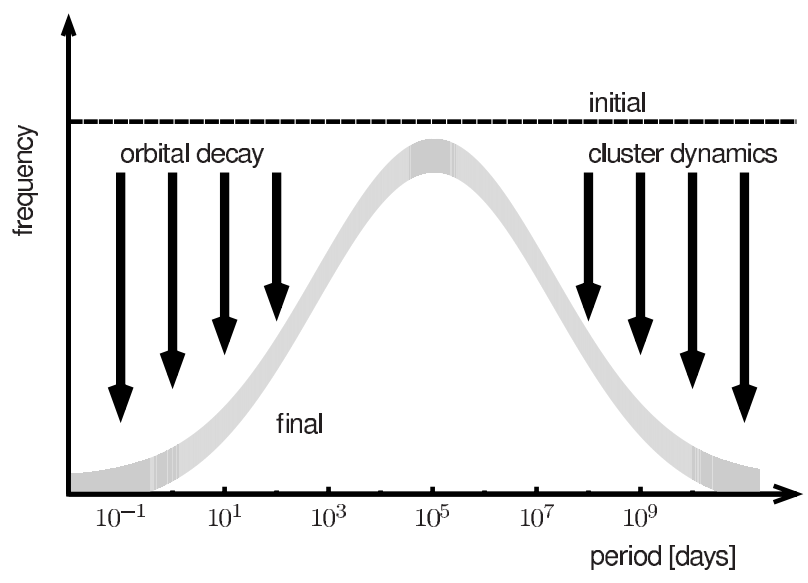

Fig. 8. Schematic illustration of the two-stage process forming the log-normal field-binary period distribution observed today (grey line) from an initial log-uniform distribution (solid line).

tribution resembles, in all its properties, remarkably that observed in the field, without any need for further assumptions. Performing a $\chi^{2}$-test of the resulting distribution and recent observations of the field binary population (Raghavan et al. 2010) yields a probability of $94.1 \%$ that both distributions originate from the same data set.

The emerging picture can be summarised in the following way (illustrated in Fig. 8): binaries born in dense, embedded clusters with initially log-uniformly period distributions are processed in two ways. As long as the cluster is embedded in its natal gas (about $1 \mathrm{Myr}$ ), the orbital decay of the embedded binaries depopulates the left-hand side of the period distribution. The dynamical evolution of the cluster destroys wide binaries, depopulating the right-hand side of the period distribution. The combined effect of these equally important processes is that the final period distribution of the binary population in the star cluster has become log-normal, although it was initially log-uniform.

This re-shaping of the period distribution leads to a reduction in the binary frequency by $29 \%$. The gas-induced orbital decay shrinks the separation between two stars such that mergers in $14 \%$ of all cases are possible. The dynamical interactions destroy $15 \%$ of the G-type binaries by means-of threebody encounters. This latter fraction only represents a lower limit to the reduction in the binary frequency owing to our specific setup procedure and can be as high as $28 \%$. Consequently, the overall reduction in the binary frequency could be as high as $42 \%$. For solar-type stars, when we assume that most stars form in an ONC-like cluster and adopt a field binary frequency of G-type primaries of 58\% (Raghavan et al. 2010), our results strongly favour a much higher initial binary frequency of $87-100 \%$, assuming instead a field binary frequency of $44 \%$; one gets $73-86 \%$.

Mass segregation and high densities in the cluster centre favour the merging of massive stars. Following the merging of predominantly massive stars, one would expect to see a difference in the IMF of single and binary stars, with the single star distribution having an excess of massive stars. This has not been observed, but the situation is more complex. Massive stars are also the most likely to capture a new partner. For example, mergers that form a stars of $M>20 M_{\odot}$, can occur on timescales of $10^{4} \mathrm{yr}$ (Pfalzner \& Olczak 2007). The merger product and its companion should then become part of the binary population again, hence the IMF of the single and binary populations should both be changed by the merging processes. Whether they would 
be affected to the same degree needs further investigations, but given the low quality statistics at the high mass end, they are indistinguishable.

The field population is clearly a mixture of stars originating from stars that experienced star formation in isolation, sparce (Taurus-like), dense (ONC-like), and very dense (Arches-like) clusters. On the one hand, associations such as the Taurus clusters probably never had a gas density above $10^{4} \mathrm{~cm}^{-3}$ and the current stellar density is below 10 stars $\mathrm{pc}^{-3}$ (Luhman et al. 2009). This means that in these systems the binary population is affected by neither orbital decay nor orbital destruction in a significant way and is therefore nearly unaltered from its primordial state. On the other hand, orbital decay becomes important for high gas densities and dynamical disruption for high stellar densities.

On the basis of actual observations, one can only speculate how many stars are born in these different density regions. While Bressert et al. (2010) stated that the minority of all stars in the solar neighbourhood form in high density regions, it is unclear whether this is also true for all of the galaxy. Dukes \& Krumholz (2011) concluded that $1 / 2-2 / 3$ of all stars are in clusters consisting of more than 1000 stars. As such massive clusters initially had much higher stellar densities (Pfalzner 2009), this indicates that environmental effects are important for these clusters. The remarkable resemblance of the distribution, obtained after both processes have taken place, to the log-normal period distribution of the field binary population is a strong indication that ONC-like clusters might be a dominant contributor to the field distribution.

In addition to the ONC-type clusters studied here, further investigations should include a variety of initial conditions: different stellar and gas density distributions (Kroupa 1995b; Parker et al. 2011), a range of cluster densities (Olczak et al. 2010; Marks et al. 2011), and different virial states of the cluster (Allison 2009). We are presently testing the limitations of our model of orbital decay using numerical simulations (Korntreff \& Pfalzner, in prep.).

To verify observationally our initial conditions, detailed studies of binary populations in very young ( $<100000 \mathrm{yrs}) \mathrm{em}-$ bedded star clusters, before the onset of the aforementioned investigated processes, would be necessary. However, any conclusions about the binary distribution in these young systems would be biased by small number statistics. Inly a few tens of stars are usually observable, although the true membership might be considerably higher owing to the high extinction in these clusters. Combining data for different clusters may help to improve the quality of our statistics. An alternative approach would be to observe different regions within a single OB associations. The processes operate predominantly in the association centre and much less so in its outskirts. Therefore, we would expect the period distribution in the association centre to differ considerably from that in the outskirt, i.e. to be close to the log-normal at the cluster centre and log-uniform in the outskirts. However, this picture neglects any mixing within the cluster, so that further theoretical work would be needed to incorporate this process.
Acknowledgements. We thank the referee for providing constructive comments and help us to improve the contents of this paper and P. Kroupa for useful discussions on this topic. The simulations were performed at the Jülich Supercomputer Centre, Research Centre Jülich within Project HKU14.

\section{References}

Allison, R. J., Goodwin, S. P., Parker, R. J., et al. 2009, ApJ, 700, L99 Binney, J., \& Merrifield, M. 1998, Galactic astronomy (Princeton University Press)

Binney, J., \& Tremaine, S. 1987, Galactic dynamics (Princeton University Press) Bonnell, I. A., \& Davies, M. B. 1998, MNRAS, 295, 691

Bressert, E., Bastian, N., Gutermuth, R., et al. 2010, MNRAS, 409, L54

Connelley, M. S., Reipurth, B., \& Tokunaga, A. T. 2008, AJ, 135, 2526

Dukes, D., \& Krumholz, M. R. 2011, ApJ, accepted [arXiv: 1111.3693]

Duquennoy, A., \& Mayor, M. 1991, A\&A, 248, 485

Fischer, D. A., \& Marcy, G. W. 1992, ApJ, 396, 178

Heggie, D. C. 1975, MNRAS, 173, 729

Hillenbrand, L. A. 1997, AJ, 113, 1733

Hillenbrand, L. A., \& Hartmann, L. W. 1998, ApJ, 492, 540

Jones, B. F., \& Walker, M. F. 1988, AJ, 95, 1755

Kaczmarek, T., Olczak, C., \& Pfalzner, S. 2011, A\&A, 528, A144

Köhler, R., Petr-Gotzens, M. G., McCaughrean, M. J., et al. 2006, A\&A, 458, 461

Kouwenhoven, M. B. N., Brown, A. G. A., Zwart, S. F. P., \& Kaper, L. 2007, A\&A, 474, 77

Kouwenhoven, M. B. N., Goodwin, S. P., Parker, R. J., et al. 2010, MNRAS, 404, 1835

Kraus, A. L., \& Hillenbrand, L. A. 2007, ApJ, 662, 413

Kroupa, P. 1995a, MNRAS, 277, 1491

Kroupa, P. 1995b, MNRAS, 277, 1522

Kroupa, P. 2000, New A, 4, 615

Kroupa, P. 2007 [arXiv: astro-ph/0703124]

Kroupa, P., \& Bouvier, J. 2003, MNRAS, 346, 343

Kroupa, P., \& Burkert, A. 2001, ApJ, 555, 945

Kroupa, P., Aarseth, S., \& Hurley, J. 2001, MNRAS, 321, 699

Lada, C. J., \& Lada, E. A. 2003, ARA\&A, 41, 57

Luhman, K. L., Mamajek, E. E., Allen, P. R., \& Cruz, K. L. 2009, ApJ, 703, 399

Marks, M., \& Kroupa, P. 2011, MNRAS, 417, 1702

Marks, M., Kroupa, P., \& Oh, S. 2011, MNRAS, 417, 1684

Moeckel, N., \& Bate, M. R. 2010, MNRAS, 404, 721

O’Dell, C. R., Henney, W. J., Abel, N. P., Ferland, G. J., \& Arthur, S. J. 2009, AJ, 137, 367

Olczak, C., Pfalzner, S., \& Eckart, A. 2010, A\&A, 509, A63

Olczak, C., Spurzem, R., Henning, T., et al. 2011, Stellar Clusters \& Associations: A RIA Workshop on Gaia, Proc., eds. E. J. Alfaro, A. T. Gallego Calvente, \& M. R. Zapatero Osorio, 142

Padmanabhan, T. 2001, Theoretical Astrophysics, Vol. 2, Stars and Stellar Systems

Parker, R. J., Goodwin, S. P., Kroupa, P., \& Kouwenhoven, M. B. N. 2009, MNRAS, 397, 1577

Parker, R. J., Goodwin, S. P., \& Allison, R. J. 2011, MNRAS, 418, 2565

Pfalzner, S. 2009, A\&A, 498, L37

Pfalzner, S., \& Olczak, C. 2007, A\&A, 475, 875

Preibisch, T., Balega, Y., Hofmann, K., Weigelt, G., \& Zinnecker, H. 1999, New A, 4, 531

Press, W. H., Teukolsky, S. A., Vetterling, W. T., \& Flannery, B. P. 2007, Numerical Recipes, The Art of Scientific Computing, 3rd edn. (New York, NY, USA: Cambridge University Press)

Prosser, C. F., Stauffer, J. R., Hartmann, L., et al. 1994, ApJ, 421, 517

Raghavan, D., McAlister, H. A., Henry, T. J., et al. 2010, ApJS, 190, 1

Reipurth, B., Guimarães, M. M., Connelley, M. S., \& Bally, J. 2007, AJ, 134, 2272

Sridharan, T. K., Beuther, H., Saito, M., Wyrowski, F., \& Schilke, P. 2005, ApJ, 634, L57

Stahler, S. W. 2010, MNRAS, 402, 1758 